AperTO - Archivio Istituzionale Open Access dell'Università di Torino

The Limits of Digital Interpretation: Semantic Versus Syntactic Connectedness

This is a pre print version of the following article:

Original Citation:

Availability:

This version is available http://hdl.handle.net/2318/1740382

since 2020-06-04T15:11:23Z

Published version:

DOI:10.1007/s11196-020-09726-5

Terms of use:

Open Access

Anyone can freely access the full text of works made available as "Open Access". Works made available under a Creative Commons license can be used according to the terms and conditions of said license. Use of all other works requires consent of the right holder (author or publisher) if not exempted from copyright protection by the applicable law. 


\title{
1 The Limits of Digital Interpretation: Semantic Versus 2 Syntactic Connectedness
}

\author{
Massimo Leone ${ }^{1,2}$ (D) \\ (c) Springer Nature B.V. 2020
}

\begin{abstract}
The concept of reasonability is key in Umberto Eco's interpretive semiotics, where it enables the formation of a community of interpreters that avoids both extremes of fundamentalism and anarchy. Such concept, however, is not immune from the technological infrastructure in which interpretation takes place. In the digital sphere, the notion itself of community is deeply altered as a consequence of fundamental change in the very nature of connectedness and connections among members.AQ1 Whereas in the pre-digital world, semantic communality would ground connectedness and the ensuing communities, in digital social networks syntactic communities prevail, where clusters of members emerge out of contagion and memetic force more than through sharing of actual semantic content. The passage from semantic to syntactic connectedness deeply affects the nature of communities and the ways in which they find cohesion. In the digital world, communities are not only syntactic more than semantic, but also quantitative more than qualitative, and negative more than positive: they take shape around what they oppose, more than around what they propose. The market is a fundamental force behind the technological framework of such new communities, since it engineers them so as to both monitor them and profit by their constant litigiousness.
\end{abstract}

Keywords Reasonableness · Interpretation · Community · Digital communication · Social networks

\footnotetext{
A first version of this article was presented as a plenary talk in the frame of the symposium "Conceptual and Normative Underpinnings of Religion \& Innovation Discourses: Social and Cultural Perspectives", Bruno Kessler Foundation, Trento, Italy, 25-26 January 2018; I thank the Director of the foundation, Marco Ventura, as well as the organizers, including Boris Rähme, for the fruitful opportunity. The final version of this article is part of a project has received funding from the European Research Council (ERC) under the European Union's Horizon 2020 research and innovation program (Grant Agreement No. 819649_FACETS).
}

Massimo Leone massimo.leone@unito.it

1 Shanghai University, Shanghai, China

2 University of Turin, Turin, Italy 


\section{Introduction}

Digital communities might seem-and are frequently presented by populisms - as freer than non-digital communities, but they are not. A democratic state parliament debates both on its laws and on the rules that are chosen as a framework to bring about such laws; digital parliaments, instead, let all-and not only their representatives-discuss about everything, except about the digital framework of discussion itself, whose inner laws are actpolly invisible and untouchable to most. Perhaps, in the future, digital communities $\_$will express a proper semiotic arena, in which not only meaning as content is discussed and negotiated, but also meaning as framework. The current evidence about such process of negotiation, however, is more than discouraging. Digital communities let unreasonableness proliferate through various forms of contagion in which no kind of negotiation plays any role. The most diriment difference between traditional communities and current digital communities, indeed, is that the latter seem to be completely uninterested-unlike the former-to any sort of community memory. They live in an eternal present that constantly flees toward a future but does not leave any traces if not in some remote servers, unexplored by anyone, or in 'time-machines' that produce no collective discourse.

Above all, no ritualization of memory exists in digital communities, as well as no established pattern to transform past interactions into guidelines for future negotiation. A community without a structured memory uneasily gives rise to stable frameworks of interpretive reasonableness, exactly insofar as these frameworks must transcend the individual agency of intentional contributors and emerge, on the contrary, from the holistic functioning of the semiosphere. We do not abide by the grammar of our natural language because someone or somewhat explicitly decided so, but because myriads of micro-interactions, including those of our ancestors, have been deposited and distilled into a configuration that, despite the possibility of micro-variations, changes, and playfulness, a community has come to accept as its standard. Unfortunately, at least thus far, such holistic mechanism of formation of a cultural memory seems not to take place in digital communities, which are constantly swept by the wind of the present.

\section{Mystical Stereotypes}

"To be connected with the whole": what does it mean? This or similar sentences often occur in the discourse of various present-day spiritual trends. They circulate through the contemporary culture by means of sundry texts and media. Even more significantly, they turn into the many stereotypical fragments through which society constructs its everyday discourse. "I feel connected with the whole" is a phrase that is often heard during night conversations among friends over a glass of wine or similia. It is necessary to investigate both the sociocultural and the semiotic meaning of affirmations of this kind, aiming at a more encompassing critique of the concept itself of connectedness, for it is instrumental in order to understand the nature of the community that the current digital sphere brings about. What does it actually mean, 
"to be connected"? Delving into the semantic field of connection, connectedness, connectivity, interconnectedness, etc. is essential in an epoch in which the terms derived from it often constitute the linguistic and textual cornerstones of the prevailing rhetoric of our times, a rhetoric according to which there would be a value in the passive status of "being connected" as well as in the active status of "connecting".

The spiritual utopia of a permanent state of connectedness with the whole is not an invention of the era of digital social networks: in the past, human beings have expressed wishes and projects of total union of a similar kind in relation to transcendence, to nature, or to humanity as a whole [1]. The concept, feeling, and rhetoric of connectedness that was formulated in relation to these different targets of complete unification were not constant but changed in relation to both the subject and the object of fusion. As a consequence, cultural semiotics must underline both continuities and discontinuities between these epochs and the current one: on the one hand, a subtle thread links the contemporary youngster that today, often stimulated by substances of various kinds, expresses a wish of total connectedness and, on the other hand, seventeenth-century Spanish Catholic mystics voicing a similar desire of merging into the totality of God [2], or the recurring utopias for a universal language [3].

On the other hand, though, the two expressions significantly differ not only as regards the specific object and context of the connectedness that is wished for, but also and most significantly, as regards the discourse that promotes, describes, and, sometimes, performs such fusion. In a nutshell, if the rhetoric of total connectedness has existed for a long time, and expressed itself first and foremost in some spiritual trends in world religions - and later in the naturalistic utopias of Romanticism and post-Romanticism - the era of digital social networks has pushed this cultural trend to a both superior and inferior level: while turning mystical connectedness into a matter of technical algorithms, accessible to all, it has also placed this anthropological drive of human beings into a marketing framework [4].

\section{The Meaning of Connectedness}

The essay that follows, then, works both at a diachronic and at a synchronic level. It retraces the current 'connectedness fever' to previous similar sociocultural manifestations in human history, and simultaneously seeks to develop a synchronic typology of its discourses. Botb aperations must start from a semiotic reflection on what a connection is. The ety $\square$ ogy of words is not equivalent to their semantics: the history of a word to its present usage but does not fully explain it, for the simple fact that speakers often ignore the history of words when they use them and sometimes even use them with a semantic content that contradicts their history. The etymology of a word, however, offers clues about the main stages and switches marking its cultural history [5]. Most probably, the etymological core of the word " " is the proto-Indoeuropean root "*ned-", which is conjectured to have covered the whole semantic area of "binding together". This root can be derived from words such as the Sanskrit "nahyati", meaning "binds, ties"; the Latin "nodus", meaning 
"knot"; the Old Irish "nascim", meaning "I bind, I oblige"; and the Old English "net", meaning "netting, network".

The same kernel of meaning occurs in all these expressions, constituting, therefore, the semantic specificity of the abovementioned proto-Furppean root. Such semantic kernel seeks to evoke a range of possible phenomend les, which in turn constitute the perceptible manifestation of a basis of ontological situations. Here follows a formal description of the main features of both this ontology and the phenomenology to which it gives rise. First, there is no connection in absolute singularity. In order for one of the phenomenological situations inscribed in the root "*ned-" to take place, ontology must be posited as dual: there are at least two elements in reality, and these two elements appear as separated. The appearance of this separation, nevertheless, deserves further reflection. For two entities to manifest themselves as distinct, indeed, a potential of connectedness must already somehow linger between them. In other words, when two beings are thought of as separated, the thought itself of separation contains a potential for interconnection. Thinking at two separate entities at the same time is inevitably tantamount to envisage at least the negative possibility of their connection. "*Ned-", therefore, refers to this idea of two or more separate entities whose separation is, however, perceived as temporary or, at least, not intrinsic.

\section{Expansions and Contractions}

From the perspective of cultural semiotics, it is especially crucial to consider how, in different epochs and societies, the range of connectedness expands or contracts according to forces that are both material and symbolical. Connecting two countries divided by a mountain range, for instance, was unconceivable for a long period of human history. In the presence of a frontier constituted by a mountain range, the two human communities separated by it were doomed to exist separately. The idea of their connection was not even envisageable, and any attempt at overcoming the gigantic obstacle mostly expressed itself in the individual endeavor of an exploration, or in the aggressive initiative of an invasion [6]. Only the development of complex technical means in late modernity (mainly from the 1830s on) allowed human beings to 'pierce' mountains and to establish permanent connections between two hitherto separated societies [7]. The engineering of tunnels not only allowed previously separated communities to interact on a regular basis, but also deeply transformed the ontology of a mountain range. People divided by mountains are cultural monads, but societies whose separation already invokes an effort of interconnection.

The same goes for other natural obstacles separating societies and, therefore, cultures: the sea was, up to modern times in human history, an agent both creating and preserving a semantics of unredeemable separation: that which was beyond Hercules's Columns could not be possibly bridged, it was immune to any human project of connection [8]. Yet, technical advancements in the art of navigation transformed the phenomenology of separation by which the planet would appear to most of its 
inhabitants: today, no sea is seen anymore as an agent of permanent seclusion. Given the appropriate navigation means, every shore in the world can be reached.

The expansion and the contraction of both the ontology and the phenomenology of potential connectedness does not depend only from material and technical circumstances. For instance, human beings do not have the technical means for reaching distant astronomic destinations yet they do not, for that matter, currently cease to perceive them as potentially reachable [9]. Since the "giant step in humankind" of the moon-landing, the space is not seen as an obstacle anymore, as something that will always keep human beings apart from what is "out there", but rather as a porous interspace, which advancement in technology will sooner or later be able to 'pierce' and 'bridge' as it was the case for mountains and oceans. Similarly, the way in which cultures stress rather the singularity of entities or their separation, and therefore their possible connection, responds also to a symbolical logic that is underpinned by material conditions but it is not completely determined by them. The interdependence between the materiality and the rhetoric of connection can also turn into independence, to such an extent that the former can actually progress to the detriment of the latter.

To give an example: technical developments in the flight industry and especially in the algorithms of pricing have led to the flourishing of low-cost flight companies, and to the consequent exponential increase in the number of journeys across country frontiers in Europe and elsewhere. Cities that would heretofore been perceived as very distant in terms of space and traveling costs have become reciprocally approachable: young people of Madrid can now easily travel to Istanbul and vice versa. On the one hand, this technical and commercial development was already prepared by some cultural conditions: the establishment and the successful running of ERASMUS, the European program for students' exchange, for instance, had already prepared the sociocultural conditions that were subsequently matched by the technology and marketing of low-cost flying.

On the other hand, though, the material conditions of connectedness and the corresponding symbolical discourse do not always develop in parallel; while this essay is being written, for instance, Europe continues to cultivate an utopia of connection, and technical meanings to ensure progress toward such utopia are in constant improvement: the current generation of European young students, for example, is able to express itself in English as European lingua franca like no other previous generation of students. At the same time, one also gathers the impression that technicalities of interconnectedness increasingly acquire a negative connotation in the light of a symbolical discourse that, opposing the former, tends to recreate an idea of separation that cannot be bridged, of a separation that inexorably slides toward a semantics of singularity and incommensurable distinction.

It is hard to determine what forces in the history of human cultures provoke the blossoming of utopias of connectedness and what other forces, on the contrary, push them toward an opposite idea of isolation and, ultimately, toward the feeling of being encircled by an incomparable, impenetrable cultural enclave. It cannot be denied, in any case, that this dialectics of expansion and contraction of the idea of connection exists, and unfolds according to patterns that are usually not linear but entail a number of paradoxical movements and convolutions. 
To recapitulate, the semantic range condensed into the proto-Indoeuropean root "*ned-" refers to the ontological existence, and the consequent phenomenological appearance, of two or more entities, which are separated, but whose separation already potentially hints at the possibility of their connection, that is, at the possibility of somehow bridging the gap that creates the separation itself. The perception of the transient character of the separation, which coincides, symmetrically, with perceiving the foreseeable character of the connection, depends on both the ontological and the phenomenological level.

\section{Ontologies and Phenomenologies of Connectedness}

At the ontological level, only a change in the material conditions of the separation allows the two or more distinct entities to be perceived under a different light, that is, under the light of a possible reconnecting. To give an examnje, the human voice was considered as having a range of $\mathrm{d} \_$ion essentially li_ ed by the power of amplification (through horns, minarets, or acoustic devices or settings) until the technological practice of its recording became viable and, between the $19^{\text {th }}$ and the $20^{\text {th }}$ century, current. From that moment on, the human voice has not been conceived anymore as inextricably imprisoned in the geography of a body, but detachable from it and able to be transmarted in a completely different anoge and time. Today, there are no more limits for t $\_$uman voice and its contents $\square$. pressions and emotions to be transplanted a distant space and (with the limitation that only the future, and not the past, is open to this transportation: we cannot send voices back to the past).

At the phenomenological level, however, the transient character of a separation is seen somehow in relation to the ontological level, but with a certain degree of independence. A separation that is ontologically transient can appear as permanent, and vice versa. The first mismatch was already exemplified through reference to interstellar traveling: probably, the technical conditions necessary to reach some remote areas of the universe will never be at the disposal of human beings; despite this ontological limit, due to such rocky facts as, for instance, the speed of light or other physical conditions, nothing can prevent human beings from dreaming about reaching, through imaginary technologies, the limits of the universe, and see with their eyes the beginning of it.

On the opposite, two entities that are perfectly bridgeable from the ontological point of view, through as simple a technology as a bus, for instance, can become phenomenologically very dictant when a symbolical discourse of distinction and singularity seeps into their p_ption. The current disintegration of the idea of a unified Europe, for is certainly not due to the ontology of geographic and linguistic frontiers: we dispose of translation machines that perform increasingly well; our trains and planes are faster and cheaper than ever. Nevertheless, despite this ontology of connectedness, and perhaps paradoxically also because of it, a discourse of singularity starts to take momentum in the gap between spaces and societies: "we shall never understand them"; "they shall never be like us"; an agency of disconnection inevitably ensues, and seeks to transform the ontology of the relation so as to be 
in line with the phenomenological perception of it: ultimately, the reestablishment of passport controls among countries of the Schengen area comes down to that: we perceive ourselves as non-connectable, therefore we must disconnect us.

\section{Agencies of Connectedness}

Along this line, it is time to formalize that which has continuously been hinted at in the last paragraphs, that is, the idea that, in the semantics that is enshrined by the proto-Indoeuropean root "*ned-", there is not only the possibility of a dialectics between the concept of a bridgeable separation and that of an unredeemable singularity, a dialectics that evolves through expansions and contractions as well as through a complex intertwining of matter and discourse, structural conditions and symbolical connotations; the semantics of connectedness also contains a fundamental idea of agency [10]. "*Ned-" and its derivatives indicate that two separate items exist, and that they can be reconnected at both the ontological and the phenomenological level, but also that a force is necessary in order for this change in the level of connection of things to take place. "Nahyati" ["binds, ties"]; "nodus" ["knot"]; "nascim" ["I bind, I oblige"]; "net", ["netting, network"]: in all old Indo-European languages, the idea emerges that, through the exertion of an appropriate agency and its force, the separateness of things can be transformed, so that two or more entities that were hitherto far from each other become closer or even merge into a mystical fusion.

The semantics of this connecting agency (which is contrasted, throughout history, by an opposite semantics of disconnecting agency) comprises two versions: in the radical warsion, the human agency is such that it can affect directly the ontology of a separat $\square$ according to the idea that this separation is actually not such at a deeper level, and therefore calls for an effort that realigns the phenomenology of things to their 'real' ontological substratum. This complex philosophical formula grasps phenomena that are indeed quite common, such as the utopia, quite frequent in certain historical periods, according to which the apparent variety of human beings is nothing but a superficial, super-structural feature, underneath which a common ground can be found. The second and less radical version does not posit a commonality of separated entities at an ontological level, but implicitly claims that, in the way they appear, that is, at the level of their phenomenology, an agency can be exerted so that previously distinct items might look as united or, at least, as closer.

\section{Grounds of Connectedness}

In both versions, a semiotic perspective on separation spontaneously arises. Even in the assumption of the separation of two or more things, indeed, a semiotic logic must somehow take place, and indicate to the beholder according to which particular angle the connection might take place. That which is here analytically exposed is actually characterized by synthetic simultaneity: when we consider two entities as separated, and when we plan and then establish a connection, we are implicitly 
looking at these two entities as signs of such connection. Framing them according to Peirce's semiotics is enlightening: entities are seen as objects in relation to which the idea of a connection selects a ground, on whose basis objects are, then, turned from static ontological items into dynamic objects, that is, into the origin of a phenomenology that is subsequently grasped by the semiotic agency of an interpretant.

The two versions described above differ because the former considers that the connection takes place at the level itself of the grounds, wharaas the latter esteems that the connection is rather established at the level of the $i$ pretants. To give an example: in the marketing of the cell phone industry first; and then in that of social networks, company mottos and advertising slogans have often emphasized the value of connection. Nokia's motto used to be "Connecting People"; Facebook's slogan is "Facebook helps you connect and share with the people in your life". In both sentences, human beings are not considered with attention to their unbridgeable singularities but as objects (in the Peircean sense of the term) from which the marketing discourse of these two companies selects a specific ground, that of the possibility and potentiality of interconnectedness. The slogans do not say what in particular, in human beings, they are going to connect. They do not specify whether, for instance, they are going to connect people with the same skin color, or with the same gender, or with the same political ideology. They more generally posit the universal interconnectedness of human beings, the fact that they are ontologically inclined to connection and that these two technological and commercial endeavors, a cell phone company and a social network service, will allow them to express this natural inclination.

These and similar slogans do not claim, like certain political ideologies of the past would do, that human beings are all equal, which was asserted by Enlightenment and the French Revolution at the civil and political level and by Marxism at the social and economic level. Political agendas of unity would emerge from such ideologies, while the ideology of connectedness implicitly suggests that human beings are actually different but also that the separatedness among them can be somehow bridged through the power of technology. Both Enlightenment and Marxism would advocate for a semantic reunification of the humankind, in terms of either juridical or economic discourse; the marketing of cell phones and social network services, on the contrary, preaches a syntactic reunification of the humankind, in which no specific content is attributed to the connection among people.

\section{Rhetorics of Connectedness}

Another rhetorical feature of these and germane slogans that is worthy of consideration is their tendency to hide or downplay the energy that the connection requires. In both the abovementioned slogans, the image takes shape of a connection that naturally establishes itself among people, as if they were spontaneously predisposed to that. The discourse of these slogans captures the essence of human beings as signs whose ground is the incoercible drive to get together. Both slogans, however, fail to emphasize that which is implicit in the semantics of the proto Indo-European root "*ned-": establishing a connection requires both an agency and an effort. Most 
derivatives of this root, indeed, refer to material or metaphoric devices of netting, such as knots or binding contracts. In the currently prevailing rhetoric of the network, instead, the knots disappear; that which remains is the result of knotting, the effect of this binding agency, force, and effort.

Refocusing on the knots, rather than on the network, allows one to retrieve two essential phenomenological features of the former: first, a connection is not only a spontaneous dynamic, caressing the natural inclination of things toward each other, but also a form of coercion, repressing the sometimes equally spontaneous tendency of things to separate from each other. The joyous marketing of social network services highlights the sparkle of the connection, as a result of which hitherto separated human beings magically start being part of a relation; this marketing, however, implicitly conceal a fundamental feature of every connection: it takes a lot of effort to keep human beings together, and such an effort cannot be uniquely syntactic, it must be a semantic one. It must, in other terms, downward explore the several layers of the human existence of an individual in order to ascertain if and when it can establish a connection with another human being, and whether this connection can be considered a more or less permanent one.

This argument brings us back to the beginn 1 of this essay, that is, to the popularity of such expressions (and ideas) as of 'total interconnectedness' or 'being connected to the whole'. When such or similar clichés of present-day mysticism pop out in casual bar conversation, the suspect arises that they are not actually prompted by familiarity with some sort of philosophical or religious holism, but that they are rather the new-age counterpart of the rhetoric of connection underpinning the marketing of social networks. In philosophical holism, instead, the idea-and the corresponding feeling — of 'being connected with the whole' stems from an excruciating study of both the whole and the self. The kind of holism that Buddhism encourages, for instance, is deeply based not on an abstract, syntactic idea of connection, but on a specific and semantic realization of suffering as binding link among all living and non-living creatures: I feel part of a whole encompassing the entire universe because a long and painful reflection has led me to realize how all beings share the same feature, from rocks to mammals, and such feature becomes the ground through which all these beings turn into as many rings of a common semiotic chain. Similarly, in Christianity, the connection that binds a community together is not generic but based on the idea of a sacrifice of oneself that, after the image of the supreme sacrifice of God-Christ, allows the community itself to take place.

Social network mysticism, on the contrary, albeit claiming for itself a quasi-m pedigree, is not a semantic but a syntactic one. It is not based on a thorough examination of the world and on the discovery of a level at which it is all underpinned by the same logic but relies on the evidence of a purely syntactic connection. Lacking any semantically specific substratum, the idea of connection that expresses itself in digitally-inspired mysticism is essentially empty. I feel connected to the whole, but I could not explain how. Two pernicious ideological features emerge from this pseudo-mystical posture: first, the ideology of total connectedness shares many of the features of the ideology of total analogy, of that 'analogical demon' that deconstructionism has somehow embodied and that semiotics has sought to criticize and unmask in all its guises. Second, both ideologies (total syntactic 
interconnectedness and total symbolical analogy) essentially voice a kind of hypocritical egotism: when present-day digital pseudo-mystics affirm to be "connected to the whole", the suspect arises that they are implicitly suggesting that "I am the whole, and I do not care about the rest".

\section{Conclusions: The Limits of Digital Interpretation}

An alarming increase in hoaxes, false rumors, and conspiracy theories characterizes most contemporary societies. Truth itself as supreme value in social conversation is disquietingly being replaced by other elements, such as the fun of trolling practices or the efficacy of populist rhetoric. More and more, interpreters revel in the belief that secrets are kept everywhere, and that abstruse hypotheses must be concocted so as to unveil the inner core of social reality. This trend is leading the contemporary episteme to a chaotic, non-functional condition, prone to gullibility, mistrust, and aggressiveness.

It is increasingly urgent to rationally expose the cultural mechanisms of mystification so as to underline the social need that they currently fulfil and propose viable antidotes to them. Since the 1970s, semiotics has been adopted as a discipline able to debunk the pernicious rhetoric of irrational persuasion; yet, the current state of the social and political discourse requires semiotics and the other humanities to undertake a further effort of meta-analysis: they are called to debunk not only the traditional rhetoric of the establishment, but also the unconventional rhetoric of the anti-establishment. Required to operate this constant, and at times paradoxical, double debunking, social analysts must come up with new, sophisticated tools of cultural decoding.

Increase in the number and density of connections among human beings enabled by technologies of digital and telematic communications, as well as the unprecedented speed, rhythm, and volume of contents that can be exchanged through such new networks, has led to, is bringing about, and is likely to be conducive in the future too to fruitful interactions among human beings at every level: it is easier than ever to share knowledge, emotions, and plans of actions with other fellow human beings, independently from where in the globe they have their physical abode. Examples are countless; in the domain of scholarship and academia, for instance, it has never been so easy to get in contact with fellow researchers at the four corners of the planet, interact with them through emails, social networks, and video-conferences, team together for common goals, organize communities and projects that stretch across cultural, linguistic, and national frontiers. The most important advantage that this global digital community has entailed is the possibility to test one's ideas, hypotheses, and interpretations not only within a limited circle of collaborators, but with a selected network whose members are scattered throughout the world.

If reasonableness is the potentiality of sharing meaning and interpretations within an intersubjective framework, through a common metalanguage, and according to an established methodology, as the etymology itself of the word "reasonability" suggests, then the new digital and telematic technologies of communication, including social networks, present scholars around the world with an unprecedented but 
healthy challenge, that of probing the penetration of new ideas not only in the limited semiosphere of one's restricted club of intellectual acolytes, but with the entire world. That might prove extremely refreshing for thinkers who seriously engage in the task of either constructing a deep knowledge of human universals, both in natural and in social sciences or, complementarily, building an articulated typology and taxonomy of human differences. Serious biologists and philosophers, as well as serious anthropologists and historians cannot but rejoice at the dramatically enlarged spectrum of their scholarly interlocutors.

Those academics, essentially a bunch of scholastic epigones, who, on the contrary, the new globalization of knowledge found self-indulgently engrossed in the petty exercise of provincial self-righteousness and self-entitlement, could not but launch their outcry at the shattering of previous intellectual frontiers. Many philosophers, with semioticians too among them, would previously thrive under the shelter of intellectual protectionism; their ideas would triumph and dominate, uncontested, and even be exported to intellectual colonies, often as academic counterpart of economic and political imperialism, sort of new version of old religious missions. It proved sufficient, however, to seek to globalize such petty semiotics to realize that its pseudo-universal schemata would encounter insurmountable aporias when applied to other cultures and semiospheres, or that they could simply not be translated in some distant languages, a clear sign that their concepts had resulted more from local linguistic games than from serious confrontations with the empirical arenas of societies and cultures.

At the same time, the limits of digital reasonableness cannot be underestimated either. The infrastructure itself of social networks and digital communication, as it was pointed out earlier, encourages an increasingly syntactic understanding of connectedness and connections, to the point that both the fact of being together with other people and the fact of being separated from them are valued, emphasized, and even made the object of radical political and pragmatic stances independently from the actual fundaments of such communities or immunities. The very practice of reasonability indeed implies the possibility of both federation and seclusion among human beings, but both are intrinsically related to the idea of a semantic ground. An invisible bond comes about among human beings, sometimes even solidifies into established networks, communities, and institutions, yet it is a bond that arises from the awareness of a shared ground. Distant physical points in societies start to be clustered into homogeneous areas of the semiosphere, not only at its kernel, but even at its experimental and revolutionary margins, precisely for people intentionally realize that some of the texts and discourses that they circulate in the semiosphere itself actually overlap. In other words, in the pre-digital world, the sense of commonality and community is a consequence of the realization of sharing a semantic space, of producing texts and discourses in relation to the environment in a similar way.

Yet, with the proliferation of digital social networks and the ensuing acceptance of the idea of connection and connectedness, the cause-effect relation is reversed: the creation of a syntactic cluster brings about not a semantic community, but the illusion of it. In pre-digital reasonableness, individuals would first sense that they could share part of their worldview and lifeform with others upon realizing the commonality of texts and discourses that they would inject in the semiosphere; this would 
give rise to the possibility of testing one's interpretations within the newly formed community, testing that would coincide with the spirit itself of communality, with the idea of giving up at least a modicum of one's solipsism in order to create and maintain a connection. In digital reasonableness, on the opposite, since the network precedes the realization of semantic communality, interpretations that are tested in the common arena are usually cannibalized by the solipsism and individualism of others. In this semiotic environment, only negative contents can survive, like those blind fish that thrive in the dark bottom of the oceans. In a community that results from syntactic connectedness, a feeling of bondedness is exclusively begotten by a sense of common opposition. As soon as a positive content is ventured in this syntactic arena, however, it is not received with hermeneutic sympathy but turned into a semantic scapegoat: syntactic networks, indeed, give rise to communities that survive only insofar they have an enemy, and as soon as they do not have one anymore, for instance because of the simple fact that they have defeated it, they must find or create a new one, or in the absence of such big external enemy, they keep creating internal scapegoats, whose continuous expulsion provides the only guarantee for the cohesion of the syntactic network.

The computability of connections and more and more also of the contents that they allow to spread and share, is enabled by digital technology but produces emerging effects that were not envisaged in the creation of their technical infrastructure: communities in the pre-digital world would of course, rely on various methods, strategies, and traditions to test t $\square$ internal cohesion and impact on the world; the capacity of a political group ef "filling a square", for instance, was a typical display of numeric force in the socio-political arena $v_{\text {ett }}$, attributing a precise value to such a display was complicated and subject to il. . ecisions, ambiguities, and, therefore, propaganda. At the end of a it was never clear how many participants had taken part in it. The establishment and the anti-establishment would provide different figures, through different media, and increasingly adopting visual strategies so as to prove their reckoning and disprove that of the counterpart, within a rhetorical struggle that was often unbalanced along common infrastructural hierarchical asymmetries. The only mechanism truly enabling the countability of opinion was the electoral one, although with some margins of imprecisions in case of corrupted or contested ballots.

In digital arenas, elections take place every second, although most people do not even realize that they are constantly voting. The digitalization of connections and contents allows a precise estimate of the shape and fluxes of social networks, but with a distortion that is clearly a blatant social manifestation of the Heisenberg effect. The quantification of connections and trends, indeed, influences the creation of new connection and trends, with such an impact and speed that the actual content of what is measured and computed is relegated in the background in relation to the measurement and the computing itself. In the sphere of pre-digital reasonableness, a semantic community would seek to fill an urban space, and usually a square, so as to prove its quantitative force; in the digital sphere, on the contrary, where syntactic communities are formed by contagion and memetic force more than through exchange of hermeneutic hypotheses of reasonability, squared are filled even before the emergence of a clear semantic commonality among those who physically 
participate in the demonstration. From the perspective of a pre-digital observer that is simply absurd, but from the point of view of a digital native that is commonsensical: since people are able to form large syntactic connections in the web, they can also visually complement it with the picture of their proxemic filling a physical square, although they would never be able to give rise to a political party, not even to a movement, $\square$ sometimes not even to a political platform or trend. Political in the digital arena resemble more and more to flash mobs, they are actually flesh mobs, displays of digital coordination more than outcomes of shared convictions, assemblies that worl as physical choreographies of a digital world in which people are attracted to a $\square$ up more by the contagious measurement of its figures than by the ideas that defends. Fashion, meant as shape that a culture takes in relation to random quantitative trends, becomes the main logic underpinning the creation of these flesh mobs, where the satisfaction of seeing oneself as part of a synchronized movement is actually more important than the motivations that caused it.

Although the dynamic of fashion is the most suitable one to explain how syntactic communities take place without resorting to any real hermeneutic exchange, the metaphor is also imperfect in the sense that clothes still have a materiality that individuals propose in distinctive and increasingly individual styles, whereas ideas do not have shape or color and often do not give rise to an outward apparel. In most present-day western political arenas, it is increasingly difficult to tell where one belongs, to which community of beliefs, judging from appearances. What people share in most syntactic communities, indeed, is a negative image of other communities, for instance when they adopt as enemy another ethnicity, or religion, or pre-digital political stance. So called digital haters, from this point of view, do nothing but radicalizing a trend that is customary in the entire world of digital syntactic communities: therein, people are what they oppose, not what they propose.

The negative vacuity of the current digital sphere, and its disinclination to give rise to forms of positive exchange and digital reasonableness, is provoked by the technological infrastructure of digital networks of communication but it involves a market. The technology and the market are in a regime of co-dependency, stemming from the same macro-ideology. The main reason for which no semantic communities can really take shape in the current social networks depends on the fact that their computability is part of a monetization strategy. For such or such group of opinion might be important to count their members and force, but for the framework itself what matters the most is that people keep connecting and $\square$ ng quantified. It is as though, in a democracy, the voting system was owned by a private company, which is not interested in the actual result of the vote, and might, therefore, even play impartial about it, but is systemically inclined to urge people to vote over and over again, to find ever new connections, to yield to the rhetorical force of numbers, to follow trends and counter-trends according to swirling fashions, although that might entail generating more and more scapegoats, attacked with increasing virulence.

That is the main cause of disequilibrium in the determination of digital reasonableness. As opinions and counter-opinions are heatedly proposed across the social networks, in the background lies a framework that, while formatting the exchanges, 
while counting them, while monitoring them, while attributing a statistic value to them, while selling and buying knowledge of such value in a market whose features are not transparent to those who hold and voice the opinions themselves, while performing all these tasks with seeming neutrality and impartiality, then, actually profits not from the reasonable intercourse of semantic contents for the sake of reaching a reasonable and hopefully long-lasting equilibrium but from the proliferation of connectedness itself and digital conversation therein. If politics is the domain of reasonable agreement, and law is the equilibrium that a community reaches throughout its internal struggles, what the commercial framework of the present-day digital conversation really wants is that people keep connecting, and exchanging, and forming temporary clusters of contagion. Unfortunately, in the current digital world, unreasonable disagreement is much more profitable than reasonable agreement.

\section{References}

1. Leone, Ma swom ). 2014. Semiotica dello slancio mistico. In Estasi/Ecstasy, monographic issue of Lexia, 15-1 Massimo Leone, 219-284. Roma. Aracne.

2. Certeau, Michel de. 1982. La fable mystique: XV e-XVIIe siècle. Paris: Gallimard; Engl. trans. 1992. The my tic able. Chicago: University of Unicago Press; to be compared with utopias of universal languagus, see Leone 2019.

3. Leone, Massimo. 2019. The Search for the Imperfect Language”. Semiotica 231: 105-119. https:// doi.org/10.1515/sem-2018-0051.

4. Natale, Simone, and Diana Pasulka (eds.). 2020. Believing in Bits: Digital media and the supernatural. Oxford: Oxford University Press.

5. Plotke, Seraina. 2017. Semantic trace of iocial interaction from antiquity to Early modern times: historical conversation. Engl. trans. by Lauia Radosh. Newcastle upon Tyne, UK: Cambridge Scholars Publishing.

6. Leone, Massimo. 2020. Apuntes para una semiótica de la frontera. Revista chilena de semiótica 12: $7-22$.

7. Stutz Steppacher, Daia Paco. 2013. Aly s-as process: Engaging montane Switzerland as an operating urban Ecology. Thesis (Advisor, Pium Bélanger), Master of Landscape Architecture in Urban Design. Harvard University, Graduate School of Design.

8. Attali, Jacques. 2017. Histoires de la mer. Paris: Fayard.

9. Friedman, Louis. 2015. Human spaceflight: from Mars to the Stars. Tucson: The University of Arizona Press.

10. Leone, Massimo (ed.). 2009. Actants, actors, ag .... The meaning of action and the action of meaning; from theories to territories. Monographic is $\overline{\overline{u \rho}}$ fLexia, new series, 3-4. Rome: Aracne.

Publisher's Note Springer Nature remains neutral with regard to jurisdictional claims in published maps and institutional affiliations. 